\title{
On the existence and social optimality of equilibria in a Hotelling game with uncertain demand and linear-quadratic costs 1
}

\author{
Michał Król 2
}

\begin{abstract}
This paper examines a variant of the Hotelling two-stage mill-pricing duopoly game with 'linear-quadratic' transport costs and the uniform customer distribution subject to a random shock. The demand is equally likely to be found anywhere in a fixed interval of feasible product characteristics, with the ex-post differentiation of tastes parametrized to reflect the degree of uncertainty. It turns out that, for uncertainty big enough, the presence of a linear component in the cost function no longer rules out an analytical solution to the game, which is a common problem in spatial competition models. In particular, a subgame-perfect equilibrium is shown to exist in which the firms' locations approach the socially efficient ones as uncertainty further increases, regardless of the curvature of the cost function. When the demand uncertainty reaches maximum, mill-pricing is equivalent to spatial price discrimination under the most general conditions.
\end{abstract}

Keywords: Hotelling, uncertainty, linear-quadratic costs

JEL Classification: C72, D43, D81, L13, R32

\section{Introduction}

Since the publication of Hotelling's original paper [15], spatial product differentiation has been a long debated issue in economic literature. The initial "Minimum Differentiation Principle" was overturned by d'Aspremont et al. 9]. They showed that in a location-thenprice duopoly with linear transportation costs and mill-pricing a pure-strategy subgameperfect equilibrium (SPNE) does not, in fact, exist. This is because the firms' tendency to agglomerate increases the incentive to price-undercut the rival, destroying the pure-strategy equilibrium (PSE) in prices. The outcome is completely different when the transportation costs are quadratic, i.e. a SPNE exists in which the firms maximize product differentiation.

The problem of PSE non-existence is by no means limited to linear costs. In another study [12, Gabszewicz and Thisse observed that, for a family of linear-quadratic costs, there is a range of locations with no second-stage PSE whenever the weight associated

\footnotetext{
${ }^{1}$ I would like to thank Prof. Paul Madden for all his support and Prof. Takatoshi Tabuchi for his comments on the final draft of the paper. This work was supported by the Economic and Social Research Council [grant number ES/G016321/1].

${ }^{2}$ Department of Economics, The University of Manchester, M13 9PL Manchester, UK. e-mail: Michal.Krol@manchester.ac.uk
} 
with the linear component of the cost function is positive. Anderson [2] analysed this in more detail, reporting, among others, two very sound negative results. First, a non-zero linear component of the cost specification rules out the existence of a pure-strategy SPNE. Second, in a game with "sufficiently linear" costs and mixed price-strategies allowed, there can be no symmetric SPNE in which the latter mixed strategies are confined to the non-PSE subgames off the equilibrium path.

Anderson then obtained the candidate equilibrium locations as best-responses to one another among all strategies that permit a second-stage PSE. He conjectured that for costs "not too linear" these locations form the SPNE of the two-stage game, and suggested this could be shown by deriving the mixed-strategy equilibrium payoffs in the non-PSE subgames (or putting some well-behaved upper bound on these payoffs). Unfortunately, as demonstrated by Osborne and Pitchik [20, this is an extremely difficult task even in the simple linear case. As a result, to this day neither the original Hotelling linear cost specification, nor in fact any cost function with a non-zero degree of linearity, admit of an analytical SPNE solution.

These problems no longer appear with mill-pricing replaced by spatial price discrimination (see [13] or [16]). Here, the socially-optimal locations (at the market quartiles) obtain in equilibrium, regardless of which combination of linear and quadratic costs is applied. However, this form of price discrimination is not always available and is difficult to justify when the firms compete in a space of consumer tastes. The purpose of this paper is to argue that in the absence of spatial price discrimination a substantial enough degree of demand uncertainty could play a similar role in ensuring social optimality and robustness to altering the usual quadratic transport cost specification. In other words, the much-debated contrasts between the two main Hotelling pricing schemes, as well as the two most common transportation cost functions (linear vs. quadratic), vanish for demand uncertainty sufficiently high.

Several papers have already introduced some form of demand uncertainty into a modified Hotelling setting. Balvers and Szerb [3] study the effect of random shocks to the products' desirability under fixed prices. Harter [14] examines the uncertainty in the form of a uniformly distributed random shift of the (uniform) customer distribution, where the firms locate sequentially. Other papers, such as [1], concentrate on the strategic effect of acquiring information about the demand through price-experimentation.

On the other hand, relatively few studies consider the effect of demand uncertainty in an otherwise unchanged Hotelling framework. Of those, Casado-Izaga [6] adopts the same form of uncertainty as Harter, but the duopolists locate simultaneously, prior to observing the actual customer distribution and then choosing prices. Meagher and Zauner [18] consider a similar setting, but succeed in parametrizing the support of the (uniform) random variable that shifts the customer distribution and report that demand uncertainty increases the equilibrium level of product differentiation. The current framework is almost the same, with the exception of a more general cost specification and some subtle changes, essential in establishing the analogy with price discrimination and detailed in section two 
of the paper.

It would appear that uncertainty could only intensify the problems (as discussed above) associated with the mill-pricing regime. It is more difficult to ensure a PSE not only for various location-pairs, but also for all realizations of customer demand. However, uncertainty makes it possible to follow Anderson's suggestion and put a tractable upper bound on the mixed strategy equilibrium (MSE) payoffs, in the form of the optimal monopoly profit (subject to the imposed consumer reservation price). This would be insufficient under certainty, but in the present context the non-PSE subgames are becoming more scarce when there is more uncertainty, meaning that the associated second-stage profits are less and less important for the first-stage expected payoff. Consequently, even a "generous" upper bound is eventually sufficient to show that no deviation from the candidate equilibrium locations is profitable.

As a result, a symmetric closed-form solution is obtained, based on the linear-quadratic cost function. For demand uncertainty sufficiently high, it is shown to constitute a SPNE as postulated by Anderson, i.e. with mixed price-strategies played only in the non-PSE subgames off the equilibrium path. However, Anderson's second negative result does not extend here, since the equilibrium exists for any "degree of linearity" of the cost function. Furthermore, as argued in section three, the current result also has an exclusively purestrategy interpretation.

The obtained equilibrium solution is particularly interesting, because as the demand becomes more uncertain, the equilibrium locations of the uncertainty mill-pricing game converge to the social-optimum associated with the SPNE of the certainty price discrimination model. In fact, the discriminatory pricing game is shown to constitute a limiting case of the current model under the most general conditions. In other words, demand uncertainty (asymptotically) reconciles the inconsistent outcomes of linear and quadratic costs under mill-pricing with the socially-efficient outcome of discriminatory pricing.

\section{The Model}

As in the classic Hotelling framework [15, there are two firms simultaneously choosing locations $x_{1}, x_{2} \in[0,1]$ in the first stage of the game and prices $p_{1}, p_{2}$ in the second stage, with a consumer located at $x$ minimizing the total cost of purchase $p_{i}+c\left(\left|x_{i}-x\right|\right)$ over $i=1,2$, so long as it does not exceed a finite reservation price $r$. The transportation cost function $c(\cdot)$ is linear-quadratic, i.e.:

$$
c\left(\left|x_{i}-x\right|\right)=a\left|x_{i}-x\right|+(1-a)\left|x_{i}-x\right|^{2}, \text { where } a \in[0,1]
$$

As usual, it is assumed that $x_{1} \leq x_{2}$ and the marginal production cost is zero. Furthermore, in the second stage of the game the firms are allowed to employ mixed price-strategies.

Uncertainty is introduced by assuming that the second-stage (ex-post) customer distribution is uniform on an interval $[z, z+m] \subset[0,1]$, i.e. contained in the space of feasible 
product characteristics. At the stage of choosing locations, the firms are uncertain of the exact value of $z$, which they know to be uniformly distributed on the interval $[0,1-m]$, where $m \in[0,1]$ is a parameter. In other words, the duopolists expect the demand to be uniform on a segment of length $m$, equally likely to be placed anywhere inside the space of feasible product characteristics.

It is clear that $m=1$ corresponds to the standard "certainty" case. As $m$ becomes smaller, the demand begins to vary between the states of nature. Initially the uncertainty is small, for instance, when $m=3 / 4$, the support of the customer distribution is equally likely to be anything from $[0,3 / 4]$ to $[1 / 4,1]$. However, as $m$ further decreases, the consumer tastes are gradually becoming more variable, until at $m=0$ they are completely statespecific; everyone is located at the same point, the distribution of which is uniform on $[0,1]$. Hence, $m$ may be thought of not only as the ex-post differentiation of tastes, but also as "the degree of certainty".

As mentioned earlier, the uncertainty is resolved between the two stages of the game. The usual solution to the location problem is then to consider a reduced game in which the payoffs associated with any $\left\{x_{1}, x_{2}\right\}$ are the expected values (with respect to the distribution of $z$ ) of the second-stage equilibrium profits associated with this location-pair.

Remark. The above specification of demand uncertainty also has a natural economic interpretation. To see this, suppose the distribution of consumer preferences is initially uniform on $[0,1]$. The customers then observe a signal indicating exactly what type of product is best and the firms think this suggestion is equally likely to be anything within the space of feasible product characteristics. Having observed a particular value $s$ of the signal, a consumer located at $x$ re-locates to $x+(s-x)(1-m)$. In other words, everyone moves towards the value of the signal, by a fraction $(1-m) \in[0,1]$ of the distance between the value of the signal and their original location. Consequently, people whose initial views were further from what is now suggested as best shift their preferences more than those individuals who were already close to the value of the signal.

It follows that $(1-m)$ can be interpreted as the "strength of the signal" and it is easy to check that the resulting ex-post customer distribution is uniform on an interval $[(1-m) s,(1-m) s+m]$. Since $s$ is uniform on $[0,1]$, the latter is a segment of length $m$, equally likely to be placed anywhere inside the space of feasible product characteristics (just as in the original model specification above).

The current approach can be seen therefore as taking the classic Hotelling framework and introducing a random shock/signal which distorts the original preferences in such a way that they are still bound to remain within the initial "main street" of $[0,1]$. When $m=1$, the signal's strength is zero and it has no effect at all. However, as $m$ decreases, the random signal begins to affect the the customer preferences more and more, making them more variable and state-specific. A demand more sensitive to random factors means there is more demand uncertainty. 
Relationship to existing models. The current "uniform-uniform" specification of demand uncertainty is similar to the one in [18]. The general framework from which both originate has the consumers uniformly distributed on a segment of length $m$, shifted by an also uniformly distributed shock with a value of up to $L$. Consequently, the support of the ex-post customer distribution can be anything from $[0, m]$ to $[L, L+m]$ and the space of feasible tastes (that could appear in some state of nature) is $[0, L+m]$. Using the classic ice-cream sellers example, this entails people uniformly distributed on a segment of length $m$, equally likely to be found anywhere on a beach of length $L+m$.

In fact, what determines the outcome of the model (subject to an appropriate rescaling) is the ratio $(L+m) / m$ of the length of the space of feasible tastes to the ex-post length of the market. This is also the most appropriate, invariant to scale measure of demand uncertainty in this context. It is therefore appropriate to reduce the framework to a one-parameter model, which could be done in various ways.

If we model demand uncertainty as an increase of $L$ while holding $m$ fixed (as in [18]), then more uncertainty means "spreading the beach", with an unchanged consumer cluster equally likely to be found anywhere inside its new boundaries. Of course, one could simply define $(-\infty,+\infty)$ as the space of feasible products, but another issue is that, as uncertainty increases, the SPNE product differentiation and average cost of purchase increase without a bound, approaching infinity in the limit. Consequently, any finite consumer reservation price, no matter how high, would have to be at some point exceeded. Such a specification would therefore seem appropriate to model demand uncertainty when it is "small", precisely the opposite of the present paper's focus of interest $t^{3}$

In contrast, the idea proposed here is to make the demand more or less variable while still "within the same beach". This is achieved by setting $L=1-m$, so as to fix the space of feasible tastes at the traditional unit interval. Consequently, the "uncertainty ratio" is now equal to $1 / \mathrm{m}$. Instead of "expanding the beach" to make the demand more uncertain, we keep it fixed, while reducing the length of the consumer cluster. In essence, the location of a needle in a haystack is more uncertain than that of a substantially larger object

Constraining the products/tastes to a finite Hotelling "Main Street" also has the advantage of allowing for a consumer reservation price that, despite being finite, is sufficiently high so as not to be strategically important anyway (exactly as it is assumed in the classic Hotelling model). This is not only realistic in economic applications, but will also prove crucial in establishing the equivalence with discriminatory pricing.

\footnotetext{
${ }^{3}$ Another study by Meagher and Zauner [17] considers a random shock arbitrarily distributed on a fixed interval. Although product differentiation is no longer unbounded, the model requires the variance of the shock to be small relative to the ex-post differentiation of tastes, so that no firm would ever capture the entire market in any state of nature. Consequently, this model is also valid for demand uncertainty not too big.
} 


\section{Results}

The reasoning behind the paper's main result below may be outlined as follows. For a convex transportation cost function, a PSE exists when both firms are located in the exterior of the ex-post customer distribution, i.e. when $x_{1}, x_{2} \notin[z, z+m]$. This is because profits are then concave in own price. On the other hand, if at least one firm located inside the market and the rival located close enough relative to the market's length, a discontinuity of marginal profits may occur where one firm encroaches on the other's hinterland. This creates an incentive to undercut the competitor's price at the candidate equilibrium (one satisfying the first-order conditions for profit maximizing), thus destroying the PSE. See [2] for the details.

Nevertheless, if the firms are distant enough from one another relative to $m$, a secondstage price equilibrium will exist for any $z \in[0,1-m]$. This is because for larger product differentiation a lower price is required to advance into the rival's hinterland and undercutting becomes less attractive.

Hence, the idea is first to identify the "candidate" (local) equilibrium locations, i.e. ones which are best-responses to one another among all locations that permit a purestrategy price equilibrium for all values of $z$. As $m$ decreases, in order to get close enough to the rival to start destroying the PSE in some subgames, one has to deviate further from the "candidate" locations, losing more expected profits due to increased price competition. Furthermore, even once non-PSE subgames begin to appear, they are less likely to occur for smaller values of $m$, because the chances of some firm ending up in the interior of a reduced market are lower. For small enough $m$, even when hoping to earn optimal monopoly profits in all non-PSE subgames, one will be better off at the "candidate" equilibrium position. This is formalized in the following.

Proposition 1 Consider a two-stage Hotelling duopoly with linear-quadratic transport costs (1), where $a \in[0,1)$, and demand uniform on a segment of length $m \in(0,1)$, equally likely to be found anywhere in $[0,1]$. For any non-binding finite reservation price $r$ and a small enough $m$ there exists a subgame-perfect Nash equilibrium in which the unique pure-strategy equilibrium prices are played in all subgames in which they exist, including the equilibrium path, while mixed strategy equilibrium prices are played in all the other subgames. In any such equilibrium, locations are given by:

$$
x_{1}^{*}=1-x_{2}^{*}=\frac{1+a(1+4 m)-8(1-a) m^{2}}{4[1+a-2(1-a) m]}
$$

Proof. See the Appendix.

It is interesting to view these findings in the context of the "certainty" mill-pricing model. In the linear case, originally studied by Hotelling, the best-response dynamics takes the duopolists towards the centre of the market, which led him to formulating the 
"Minimum Differentiation Principle" [15]. This was overturned later [9], by observing that this tendency to reduce product differentiation eventually makes the firms enter the area where no PSE exists.

In contrast, in Anderson's linear-quadratic model [2], corresponding to the case of $m=1$ in the current specification, one can identify candidate equilibrium locations for "not too linear" transportation costs 4 . Those locations are "local" equilibria, in the sense that a firm's second-stage PSE profit will decline as it moves away from the candidate equilibrium location and towards the rival. However, one can still locate close enough to the competitor so as to eliminate the PSE, making it impossible to show that the proposed locations form a SPNE without obtaining some estimate of the payoffs in the non-PSE subgames.

Similarly, in case of $m \in(0,1)$ studied in Proposition 1 one can identify "local" equilibrium locations such that locating any closer to the counterpart will decrease the secondstage expected profit, as long as PSE in prices exist for all realizations of the uncertainty $z \in[0,1-m]$. This can be done for $1-a$ sufficiently large relative to $m$ ("not too linear" costs) or, conversely, for $m$ small enough relative to $1-a$.

In fact, for $m$ sufficiently close to 0 , a symmetric SPNE is no longer impossible for "linear enough" costs. The candidate equilibria exist for all $a \in[0,1)$ and can be shown to form SPNE. In other words, not only does Anderson's negative result not extend here, but the SPNE with locations $x_{i}^{*}$ may be established.

The finite reservation price $r$ is instrumental in proving Proposition 11. In fact, the possibility of consumers choosing an "outside option" at some point has already been studied as a potential remedy for the problem of PSE non-existence under certainty. See, for example, Economides [10] or, for an extensive recent discussion of the resulting secondstage equilibria, consult Mérel and Sexton [19].

The difference is that the current model follows the mainstream of spatial competition literature in assuming that $r$ is "non-binding", i.e. sufficiently high so as not to affect the equilibrium characteristics in any way. Despite this, it turns out it can still facilitate establishing the SPNE. This is because the reservation price is used not as a factor affecting the players' PSE play, but rather as a basis for putting an upper bound on the MSE payoffs when the PSE fail to exist.

Such a "generous" upper bound would still be insufficient in case of $m=1$, as studied by Anderson. However, the non-PSE subgames become more scarce as $m$ decreases. Hence, any finite benefits of deviating from $x_{i}^{*}$ achieved in those subgames are eventually outweighed by the losses due to increased price competition incurred in those subgames in which the PSE still exist.

We now turn to the case of $a=1$, i.e. the standard linear cost function. This is the case most-exposed to the problem of PSE non-existence. Firstly, the incentives to price-undercut the rival at her location are maximized, since this results in instantaneously

\footnotetext{
${ }^{4}$ Although Anderson uses two parameters, $a$ and $b$ as the respective weights of the linear and quadratic components, it turns out that the results only depend on $a / b$, i.e. the model is compatible with the current specification.
} 
acquiring the entire market. Secondly, the cost function is least convex among the studied class, minimizing the tendency for strategic product differentiation, and hence making it easier to undercut the closely located competitor. For more on the relationship between the curvature of transport costs and PSE existence under certainty, see [11].

The reason why $a=1$ is a case which requires a separate treatment is that the secondstage profit functions are no longer continuous. However, the proof of Proposition 2 below is analogous to that of Proposition 1 above and the latter result can be extended here. In fact, because this case is also relatively simple in terms of mathematical tractability, more can be said about the degree of uncertainty that is sufficient for the proposed SPNE to occur.

Proposition 2 Consider a two-stage Hotelling duopoly with linear transport costs (1), where $a=1$, and demand uniform on a segment of length $m \in(0,1)$, equally likely to be found anywhere in $[0,1]$. For any non-binding finite reservation price $r$ and a small enough $m$ there exists a subgame-perfect Nash equilibrium in which the unique pure-strategy equilibrium prices are played in all subgames in which they exist, including the equilibrium path, while mixed strategy equilibrium prices are played in all the other subgames. In any such equilibrium, locations are given by $x_{i}^{*}$ with $a=1$. Furthermore it is sufficient that $m \leq 1 / 9$ for such an equilibrium to appear.

Proof. See the Appendix.

The above threshold value of $m$ is relatively low. However, there are good reasons to believe that, in general, less uncertainty is required to ensure the existence of the proposed SPNE.

First of all, in establishing the above sufficient condition no attempt was made to obtain the exact MSE payoffs in the non-PSE subgames. Instead, a "generous" upper bound was put on those payoffs, equal to the maximum of what a firm could earn over all price-pairs. This was partly with mathematical tractability in mind and partly to preserve an exclusively pure-strategy interpretation of the obtained SPNE5. Nevertheless, there is evidence to suggest that the MSE payoffs are lower than those in the candidate PSE (satisfying the first-order conditions) and diminishing quickly as product differentiation falls (see [20]). In any case, they must be significantly lower than the applied upper bound and hence less uncertainty is required to render a deviation from $x_{i}^{*}$ into the non-PSE area unprofitable.

Another reason why, in general, less uncertainty should be necessary for the proposed SPNE to exist, is that, as already indicated, $a=1$ is the case most-exposed to the problem of PSE non-existence. Consequently, the threshold value of $m$ is likely to be lower than $1 / 9$ for $a<1$, despite being more difficult to obtain.

\footnotetext{
${ }^{5}$ By construction, locations $x_{i}^{*}$ are best-responses to one another regardless of what happens in any non-PSE subgames. Hence, in a game with pure-strategy pricing, a player $i$ who considers the unique PSE a credible indication of second-stage profits will also regard $x_{i}^{*}$ as an optimal reaction to $x_{-i}^{*}$.
} 
Finally, one could consider binding (i.e. arbitrarily low) reservation prices. Although complicated on the technical side, this would allow for lower upper bounds on the MSE payoffs, while also potentially reducing the range of locations / states of nature where the PSE fail (see [10]).

A closer look at the SPNE locations (2) reveals that $\partial x_{1}^{*} / \partial a>0$. Not surprisingly, a higher curvature of the cost function results in an increased tendency for strategic product differentiation, just as it happens under certainty. However, we also have $\partial^{2} x_{1}^{*} / \partial a \partial m>0$, meaning that this effect is weakened when demand uncertainty increases. In other words, the model is more robust to variations in the transport cost function when the demand becomes more uncertain.

In fact, as $m \rightarrow 0$, i.e. approaches the extreme case all customers located at the same point, we have convergence to $x_{1}^{*}=1-x_{2}^{*}=1 / 4$ for any $a \in[0,1]^{6}$. This leads to an interesting analogy with the aforementioned 'certainty' model of discriminatory (rather than mill-) pricing.

Corollary 3 As $m \rightarrow 0$, the equilibrium locations $x_{i}^{*}$ converge to the socially optimal ones associated with the certainty price-discrimination model for any $a \in[0,1]$.

The next proposition shows that this is by no means a coincidence. In the extreme case of $m=0$, a direct equivalence between demand uncertainty and discriminatory pricing holds under the most general conditions, in terms of the distribution of the random shock, the transport cost function and the number of players, which can be greater than twd 7 .

Proposition 4 Consider a two-stage Hotelling mill-pricing n-player game with any nondecreasing transport cost function $c\left(\left|x_{i}-x\right|\right)$, in which all consumers are located at the same point drawn from a probability distribution F. This game is equivalent to a "certainty" price discrimination Hotelling n-player game with transport cost function $c\left(\left|x_{i}-x\right|\right)$ and customer distribution F.

Proof. Take a second-stage price subgame of the "uncertainty" game in which the consumers are located at point $z$. This is clearly the same as a Bertrand game with firm $i$ producing at a (asymmetric) cost $c\left(\left|x_{i}-x\right|\right)$. Consequently, the firm closest to $z$ (and hence most cost-efficient) captures the entire demand, by marginally undercutting a zero mill price of the second-closest firm. The profit of firm $i$ is therefore:

$$
\pi_{i}^{*}\left(x_{1}, \ldots, x_{n}, z\right)=\max \left\{0, \min _{j \notin i}\left[c\left(\left|x_{j}-z\right|\right)-c\left(\left|x_{i}-z\right|\right)\right]\right\}
$$

\footnotetext{
${ }^{6}$ A similar observation has been made by Kieron Meagher in the context of a quadratic cost game $(a=0)$ with uncertainty specified as in 18. This can be found in an unpublished working paper "On the Equivalence of Asymptotic Demand Location Uncertainty and Spatial Price Discrimination". I am grateful to Kieron Meagher for making it available to me.

${ }^{7}$ This refers to the usual $n$-player Hotelling framework (as in [5]). Each consumer minimizes the total purchasing cost $p_{i}+c\left(\left|x_{i}-x\right|\right)$ over $i \in 1,2, . ., n$ and the firms simultaneously choose first locations and then prices. Everything else is as defined in Section 2.
} 
which is the same as the second-stage equilibrium profit attained at location $z$ in the "certainty" price discrimination game (see [13] or [16]). Consequently, the first stage expected profit in the "uncertainty" game becomes:

$$
\Pi_{i}\left(x_{1}, \ldots, x_{n}\right)=\int_{-\infty}^{+\infty} \pi_{i}^{*}\left(x_{1}, \ldots, x_{n}, z\right) F(z)
$$

which, again, is the same as the total profit from all locations in the certainty price discrimination game. As the payoff functions and strategy spaces are the same, the two games are equivalent.

The above result provides a helpful insight into the mechanics of the model and makes it possible to interpret the first two propositions in terms of the analogy between demand uncertainty and discriminatory pricing.

When the demand varies between the states of nature, the firms are effectively price discriminating between them, adapting different price strategies for different realizations of the uncertainty. As $m$ decreases, it becomes more likely for the ex-post consumer demand to be located in a firm's hinterland, while also far enough from the rival for the firm to favour capturing it entirely. Such "monopolistic" equilibria gradually replace the "competitive" ones and the firms' profits are to an increasing extent stemming from the advantage in transportation costs, i.e. from being better placed relative to the ex-post customer demand. This creates an incentive for the duopolists to minimize the transport costs incurred by the consumers over all states of nature, which means implicitly pursuing a socially-optimal objective. For this reason, the curvature of the cost function also becomes less and less important for location decisions. As $m$ goes to zero, the equilibrium locations $x_{i}^{*}$ associated with different values of $a$ converge to the efficient ones associated with the "certainty" price discrimination model.

On the one hand, these results are in contrast with those of Meagher and Zauner ([17], [18]). Instead of an increase of product differentiation and a decline of welfare as uncertainty increases, we have convergence to the intermediate, socially optimal differentiation level as uncertainty approaches its upper bound.

On the other hand, it is interesting to note that for large levels of demand uncertainty locations $x_{i}^{*}$ are not far from the ones obtained by Osborne and Pitchik [20] who focused on the mixed-strategy price equilibria under certainty and linear costs. In a sense, demand uncertainty is similar to price-randomization, in that any strategy-pair produces a random distribution of second-stage profit allocations.

In fact, an even more direct parallel can be drawn between the current model and the work of Bester et al. [4]. The latter study considers mixed-strategy locations under quadratic costs and the resulting PSE in prices. When a player responds to a random distribution of the competitor's locations for a fixed distribution of tastes, it is similar to responding to a fixed location of the competitor when the exact placement of the customer demand is random. Either way, a firm is uncertain of how close it would be to the consumer 
distribution when compared with the rival, which is what drives the second-stage equilibrium profits. However, in the present model a firm cannot itself set a random response, but is restricted to choosing a single location. It is therefore to no surprise that the former, richer strategy space results in an infinite number of possible equilibrium configurations (see [4] for the details). What the current approach and the other two mentioned have in common is that they all allow for an intermediate level of product differentiation, possibly more realistic than the extreme maximum or minimum differentiation results.

\section{Concluding Remarks}

The paper examined the Hotelling two-stage mill-pricing duopoly with customer demand uniform on a segment of a given length, equally likely to be found anywhere in the usual unit interval of feasible product characteristics. Parametrizing the segment's length to reflect the degree of uncertainty made it possible to avoid assuming an infinite customer reservation price. This in turn allowed for an investigation of the effect of an arbitrarily uncertain demand.

In particular, based on a linear-quadratic cost function, SPNE were established for demand uncertainty sufficiently large, similar to the candidate equilibria postulated by Anderson under certainty. However, Anderson's negative results do not extend here, as the proposed SPNE exist for any degree of linearity of the cost function.

In fact, as demand uncertainty increases, the curvature of transport costs becomes less important for the equilibrium outcome and the SPNE locations associated with different linear-quadratic cost functions converge at the socially-optimal ones associated with the certainty price-discrimination model. In other words, the much-debated contrasts between the two main Hotelling pricing schemes, as well as the two most common transportation cost functions (linear vs. quadratic), vanish for demand uncertainty sufficiently high.

The intuitive explanation of those results relies upon observing that, when setting different prices for different realizations of customer demand, the duopolists are effectively price-discriminating between the states of nature. When the ex-post differentiation of tastes decreases, it becomes vital to secure the equivalent of a cost advantage over the opponent, by making it cheaper for the customers to get to the firm's location. Eventually, the flexibility of price-discriminating between the states of nature becomes close to that of setting prices independently at each physical location and the firms implicitly pursue the socially desirable objective of minimizing the total transportation costs. In the limiting case of consumer tastes being completely state-specific, the "uncertainty" mill-pricing model and the "certainty" discriminatory pricing game are equivalent under the most general conditions. 


\section{Appendix}

For shortness, some algebraic derivations are relegated to the on-line Wolfram Mathematica appendix (http://tinyurl.com/29vk5vl), where numbers in curly brackets $\{\#\}$ represent a position therein where a particular statement is verified.

Proof of Proposition 1. We begin by obtaining the "candidate" equilibrium locations (2). To this end, consider first a second-stage subgame associated with a particular locationpair and value $z$ of the random shock. Assume, for the moment, that the firms are restricted to playing pure-strategy prices and let $\widetilde{x}_{i}$ denote the value of $x$ solving equation $(\mathrm{A} i)$ below:

$$
\begin{aligned}
& p_{1}+a\left(x_{1}-x\right)+(1-a)\left(x_{1}-x\right)^{2}=p_{2}+a\left(x_{2}-x\right)+(1-a)\left(x_{2}-x\right)^{2} \\
& p_{1}+a\left(x-x_{1}\right)+(1-a)\left(x_{1}-x\right)^{2}=p_{2}+a\left(x-x_{2}\right)+(1-a)\left(x_{2}-x\right)^{2} \\
& p_{1}+a\left(x-x_{1}\right)+(1-a)\left(x_{1}-x\right)^{2}=p_{2}+a\left(x_{2}-x\right)+(1-a)\left(x_{2}-x\right)^{2}
\end{aligned}
$$

where (A1) and (A2) correspond to the hinterlands of the respective firms and (A3) to the area "in between". Next, define $p_{i . j}^{c}$ as the candidate "competitive" equilibrium price of firm $i$ in area $j$, i.e. as the solution to:

$$
\frac{\partial\left[p_{1}\left(\widetilde{x}_{j}-z\right) / m\right]}{\partial p_{1}}\left(p_{1 . j}^{c}, p_{2 . j}^{c}\right)=\frac{\partial\left\{p_{2}\left[1-\left(\widetilde{x}_{j}-z\right) / m\right]\right\}}{\partial p_{2}}\left(p_{1 . j}^{c}, p_{2 . j}^{c}\right)=0
$$

where $\pi_{i . j}^{c}$ is the corresponding candidate equilibrium profit. Similarly, let $p_{i . j}^{m}$ denote the candidate "monopolistic" equilibrium price of firm $i$ in area $j$, i.e. the solution to $\widetilde{x}_{j}\left(p_{1 . j}^{m}, 0\right)=z+m$ for player 1 and $\widetilde{x}_{j}\left(0, p_{2 . j}^{m}\right)=z$ for player 2 . For "exterior" locations, i.e. $x_{1}, x_{2} \notin[z, z+m]$, the profit functions are concave and a PSE must exist. Indeed, in case of $z+m \leq x_{1}$ we have $\{1\}$ :

$$
\frac{\partial\left[p_{1}\left(\widetilde{x}_{1}-z\right) / m\right]}{\partial p_{1}}\left(p_{1.1}^{m}, 0\right)<0 \Leftrightarrow z<z_{1.1}=\frac{1}{2}\left(\frac{a}{1-a}+x_{1}+x_{2}\right)-2 m
$$

i.e. for $z<z_{1.1}$ firm 1 chooses to take the whole market even when $p_{2}=0$, earning $p_{1.1}^{m}$. On the other hand, for $z \geq z_{1}^{1}$ a competitive equilibrium is "feasible", i.e. $\widetilde{x}_{1}\left(p_{1.1}^{c}, p_{2.1}^{c}\right) \leq$ $z+m\{2\}$. By symmetry, for $z>x_{2}$ and $z>z_{2.2}=\left(x_{1}+x_{2}-a /[1-a]\right) / 2+m$ firm 2 monopolizes the market, while for $x_{2}<z<z_{2.2}$ profits are given by $\pi_{i .2}^{c}$.

When the market is not contained in some firm's hinterland, we have $\{3\}$ :

$$
\begin{aligned}
\frac{\partial p_{1}\left[p_{1}\left(\widetilde{x}_{3}-z\right) / m\right]}{\partial p_{1}}\left(p_{1.3}^{m}, 0\right) & <0 \Leftrightarrow z<z_{1.3}=\frac{1}{2}\left(x_{1}+x_{2}\right)-2 m \\
\frac{\partial p_{2}\left[1-\left(\widetilde{x}_{3}-z\right) / m\right]}{\partial p_{2}}\left(0, p_{2.3}^{m}\right) & <0 \Leftrightarrow z>z_{2.3}=\frac{1}{2}\left(x_{1}+x_{2}\right)+m
\end{aligned}
$$


i.e. for $x_{1}-m<z<z_{1.3}$ firm 1 captures the entire market at price $p_{1.3}^{m}$, while firm 2 does the same for $z_{2.3}<z<x_{2}$ at price $p_{2.3}^{m}$. On the other hand, when the firms are placed on the market's opposite sides (i.e. $x_{1}<z$ and $z+m<x_{2}$ ) then profits are concave and given by $\pi_{i .3}^{c}$ in equilibrium for $z_{1.3}<z<z_{2.3}$. Furthermore, for $x_{2}-x_{1}>4 m$ we have $z_{1.3}>x_{1}$ and $z_{2.3}<x_{2}-m$, which means a PSE exists for any value of $z$. Suppose that, in addition, $x_{1}>m$ and $x_{2}<1-m$. Then the expected PSE profit of firm 1 is:

$$
\Pi_{1}\left(x_{1}, x_{2}\right)=\int_{0}^{x_{1}-m} p_{1.1}^{m} d z+\int_{x_{1}-m}^{z_{1}{ }^{3}} p_{1.3}^{m} d z+\int_{z_{1.3}}^{z_{2.3}{ }^{3}} \pi_{1.3}^{c} d z
$$

where we have used the fact that $z_{1.3}<z_{1.1}$ and skipped multiplying by $1 /(1-m)$, the density of the distribution of $z$, since it will not affect the optimal choice of location. By symmetry, we have $\Pi_{2}\left(x_{1}, x_{2}\right)=\Pi_{1}\left(1-x_{2}, 1-x_{1}\right)$ and $\{4\}$ :

$$
\frac{\partial^{2} \Pi_{1}}{\partial x_{1}^{2}}<0, \frac{\partial^{2} \Pi_{2}}{\partial x_{2}^{2}}<0
$$

leading to the first order conditions:

$$
\frac{\partial \Pi_{1}}{\partial x_{1}}\left(x_{1}^{*}, x_{2}^{*}\right)=\frac{\partial \Pi_{2}}{\partial x_{2}}\left(x_{1}^{*}, x_{2}^{*}\right)=0
$$

The only solution to those FOC's which could satisfy $x_{2}^{*}-x_{1}^{*}>4 m$ and $m<x_{1}^{*}<x_{2}^{*}<1-m$ is (2) and a sufficient condition for this to happen is $m \leq 1 / 10\{5\}$.

Let $\Pi^{*}$ denote the expected profit obtained by substituting (2) into $\Pi_{1}$. We will show that for $m$ small enough it is not profitable for player 1 to deviate from $x_{1}^{*}$, regardless of what the players do in the non-PSE subgames.

Consider first a deviation to $x_{1}<m$, in which case the expected profit is:

$$
\widehat{\Pi}_{1}^{1}\left(x_{1}, x_{2}^{*}\right)=\int_{0}^{z_{1.3}} p_{1.3}^{m} d z+\int_{z_{1.3}}^{z_{2.3}} \pi_{1.3}^{c} d z
$$

and we have $\{6\}$ :

$$
\frac{\partial \widehat{\Pi}_{1}^{1}}{\partial x_{1}}\left(x_{1}, x_{2}^{*}\right)>0 \text { for } x_{1}<m<1 / 10
$$

Next, consider the case of $x_{2}^{*}-4 m<x_{1}<x_{2}^{*}-2 m$, so that $x_{1}-m<z_{1.3}<x_{1}$ and $x_{2}^{*}-m<z_{2.3}<x_{2}^{*}$. Since the profits in any subgame (including the non-PSE ones) cannot exceed $r$, the overall expected profit must then be smaller than:

$$
\int_{0}^{x_{1}-m} p_{1.1}^{m} d z+\int_{x_{1}-m}^{z_{1.3}} p_{1.3}^{m} d z+\int_{z_{1.3}}^{x_{1}} r d z+\int_{x_{1}}^{x_{2}^{*}-m} \pi_{1.3}^{c} d z+\int_{x_{2}^{*}-m}^{z_{2.3}} r d z
$$


which in turn is less than:

$$
\widehat{\Pi}_{1}^{2}\left(x_{1}, x_{2}^{*}\right)=\int_{0}^{x_{1}-m} p_{1.1}^{m} d z+\int_{x_{1}-m}^{x_{1}} r d z+\int_{z_{1.3}}^{z_{2.3}} \pi_{1.3}^{c} d z+\int_{x_{2}^{*}-m}^{x_{2}^{*}} r d z
$$

where the fact that $\pi_{1.3}^{c} \geq 0\{7\}$ was used. For $m \leq 1 / 10, \widehat{\Pi}_{1}^{2}\left(x_{1}, x_{2}^{*}\right)$ is concave in $x_{1}\{8\}$. Solving:

$$
\frac{\partial \widehat{\Pi}_{1}^{2}}{\partial x_{1}}\left(x_{1}, x_{2}^{*}\right)=0
$$

for $x_{1}$, substituting the obtained value back into $\widehat{\Pi}_{1}^{2}\left(x_{1}, x_{2}^{*}\right)$, subtracting the outcome from $\Pi^{*}$ and finally calculating the limit of the resulting expression as $m \rightarrow 0$ yields $(5+7 a) / 256>0\{9\}$. This means that for $m$ sufficiently small $\widehat{\Pi}_{1}^{2}\left(x_{1}, x_{2}^{*}\right)$ is less than $\Pi^{*}$ and a deviation from $x_{1}^{*}$ to $x_{1} \in\left[x_{2}^{*}-4 m, x_{2}^{*}-2 m\right]$ is not profitable.

Next, note that for $m \in[0, a /[2(1-a)])$ we have $z_{1.1}>x_{1}-m$ and $z_{2.2}<x_{2}^{*}\{10\}$ and consider the following cases:

1. $\{11\} x_{1} \in\left[x_{2}^{*}-2 m, x_{2}^{*}-m\right]:$

$$
\widehat{\Pi}_{1}^{3}\left(x_{1}, x_{2}^{*}\right)=\int_{0}^{x_{1}-m} p_{1.1}^{m} d z+\int_{x_{1}-m}^{x_{1}} r d z+\int_{x_{1}}^{x_{2}^{*}-m} \pi_{1.3}^{c} d z+\int_{x_{2}^{*}-m}^{x_{2}^{*}} r d z
$$

and since:

$$
\frac{\partial \widehat{\Pi}_{1}^{3}}{\partial x_{1}}\left(x_{1}, x_{2}^{*}\right)<0 \text { for } x_{1} \in\left[x_{2}^{*}-2 m, x_{2}^{*}-m\right] \text { and } m \leq \min \{a /[2(1-a)], 1 / 10\}
$$

it is sufficient to observe that:

$$
\lim _{m \rightarrow 0}\left[\Pi^{*}-\widehat{\Pi}_{1}^{3}\left(x_{2}^{*}-2 m, x_{2}^{*}\right)\right]=\frac{2+a}{16}>0
$$

2. $\{12\} x_{1} \in\left[x_{2}^{*}-m, x_{2}^{*}\right]$ :

$$
\widehat{\Pi}_{1}^{4}\left(x_{1}, x_{2}^{*}\right)=\int_{0}^{x_{1}-m} p_{1.1}^{m} d z+\int_{x_{1}-m}^{x_{2}^{*}} r d z
$$

and since:

$$
\frac{\partial \widehat{\Pi}_{1}^{4}}{\partial x_{1}}\left(x_{1}, x_{2}^{*}\right)<0 \text { for } x_{1} \in\left[x_{2}^{*}-m, x_{2}^{*}\right], r>0 \text { and } m \leq 1 / 10
$$

it is sufficient to observe that:

$$
\lim _{m \rightarrow 0}\left[\Pi^{*}-\widehat{\Pi}_{1}^{4}\left(x_{2}^{*}-m, x_{2}^{*}\right)\right]=\frac{2+a}{16}>0
$$


Finally, note that in the present case of $a \in[0,1)$ the second-stage profit functions are continuous and hence, by Theorem 3 in Dasgupta and Maskin [7] a mixed-strategy price equilibrium always exists. Clearly, the corresponding profits may not exceed $r$. It follows that for $m$ sufficiently small player 1 (and, by symmetry, player 2) cannot profitably deviate from the equilibrium characterized in Proposition 1. Finally, note that the candidate equilibrium locations are unique and hence any SPNE which entails playing the PSE where available must have locations (2).

Proof of Proposition 2 2 Again, some algebraic derivations are relegated to the online appendix, where numbers in curly brackets $\{\#\}$ represent a position therein where a particular statement is verified.

We begin by obtaining the "candidate" equilibrium locations (2). Clearly, for $z<x_{1}-m$ the unique price equilibrium is $\left\{p_{1}=x_{2}-x_{1}, p_{2}=0\right\}$, i.e. firm 1 doing just enough to capture the whole market with firm 2 setting its price to zero. For $x_{1}-m<z<z_{1}^{m}=$ $\frac{1}{2}\left(x_{1}+x_{2}-4 m\right)$ firm 1 still attracts all consumers, earning a profit of $x_{1}+x_{2}-2(z+m)$ $\{13\}$. Conversely, firm 2 takes the whole market for $z>z_{2}^{m}=\frac{1}{2}\left(2 m+x_{1}+x_{2}\right)\{14\}$. For the intermediate $z_{1}^{m}<z<z_{2}^{m}$, either no equilibrium exists or there is a usual "competitive" equilibrium, obtained by finding the location of the "indifferent" consumer $\widetilde{x}$ and solving

$$
\frac{\partial\left\{p_{1}(\widetilde{x}-z) / m\right\}}{\partial p_{1}}=\frac{\partial\left\{p_{2}[1-(\widetilde{x}-z) / m]\right\}}{\partial p_{2}}=0
$$

for $p_{1}, p_{2}$, which gives the equilibrium profit of firm 1 :

$$
\pi_{c}^{*}=\frac{\left[x_{1}+x_{2}-2(z-m)\right]^{2}}{18 m}
$$

The necessary and sufficient condition for the price equilibrium to exist for all $z_{1}^{m}<z<z_{2}^{m}$ is $x_{2}-x_{1} \geq 2 m\{15\}$, which also ensures that $x_{1}-m<z_{1}^{m}$. Assume first that $x_{1}<m$. The expected profit of firm 1 is then:

$$
\frac{1}{1-m}\left[\int_{0}^{z_{1}^{m}} x_{1}+x_{2}-2(z+m) d z+\int_{z_{1}^{m}}^{z_{2}^{m}} \pi_{c}^{*} d z\right]
$$

which is increasing in $x_{1}$ for $x_{1}<m$ and $x_{2}-x_{1} \geq 2 m\{16\}$. On the other hand, for $x_{1} \in\left[m, x_{2}-2 m\right]$, the expected profit of firm 1 is given by:

$$
\frac{1}{1-m}\left[\int_{0}^{x_{1}-m} x_{2}-x_{1} d z+\int_{x_{1}-m}^{z_{1}^{m}} x_{1}+x_{2}-2(z+m) d z+\int_{z_{1}^{m}}^{z_{2}^{m}} \pi_{c}^{*} d z\right]
$$


which evaluates to $\{17\}$ :

$$
\Pi_{1}\left(x_{1}, x_{2}\right)=\frac{4 m^{2}+4 m x_{1}-3 x_{1}^{2}-4 m x_{1}+2 x_{1} x_{2}+x_{2}^{2}}{4-4 m}
$$

By symmetry of the game, we have $\Pi_{2}\left(x_{1}, x_{2}\right)=\Pi_{1}\left(1-x_{2}, 1-x_{1}\right)$. Since $\partial^{2} \Pi_{i} / \partial x_{i}^{2}=$ $3 /[2(m-1)]<0\{18\}$, the candidate equilibrium is given by $\{19\}$ :

$$
\frac{\partial \Pi_{1}}{\partial x_{1}}=\frac{\partial \Pi_{2}}{\partial x_{2}}=0 \Leftrightarrow x_{1}^{*}=1-x_{2}^{*}=\frac{1}{4}(1+2 m)
$$

which means both players earn:

$$
\Pi^{*}=\frac{3-12 m+28 m^{2}}{16(1-m)}
$$

and the condition $x_{2}^{*}-2 m \geq x_{1}^{*}>m$ is satisfied for $m \leq 1 / 6\{20\}$.

We now establish the conditions for $\left\{x_{1}^{*}, x_{2}^{*}\right\}$ to form a SPNE, by showing that no deviation to a $x_{1}>x_{2}^{*}-2 m$ can give player 1 an expected profit larger than $\Pi^{*}$ for uncertainty big enough relative to a non-binding reservation price $r$.

First of all, the smallest such price is $r=x_{2}^{*}$. To see this, observe first that for any response to $x_{2}^{*}$, no consumer ever has to incur a total cost of more than $x_{2}^{*}$ in any "competitive" price equilibrium $\{21\}$. Consequently, the associated equilibrium payoffs remain the same, while the profits from any price-deviation could only decrease with the reservation price imposed. This leaves any "competitive" price equilibria unaffected by the reservation price. On the other hand, in any "monopolistic" equilibrium the total cost incurred by a consumer cannot exceed the cost of travelling to the location of the player who sets his mill-price to zero. This means it cannot exceed $r$ when the player located at $x_{2}^{*}$ ends up with no market share. And when it is the deviating player who ends up with nothing, $r$ does not matter for his second-stage equilibrium profit anyway.

The next step is to verify that with $r \geq x_{2}^{*}, x_{1} \in\left(x_{2}^{*}-2 m, x_{2}^{*}\right), p_{2}=r$ and either firm located in $[z, z+m]$, firm 1 would choose a $p_{1}$ to capture the entire market subject to the imposed reservation price $\{22\}$. This gives an upper bound of $\bar{\pi}_{l}=\left(r-x_{1}+z\right)$ on the second stage profits of the deviating firm when $x_{1}-m<z<x_{1}-m / 2$ and $\bar{\pi}_{r}=\left(r-m+x_{1}-z\right)$ when $x_{1}-m / 2<z<x_{2}\{23\}$.

Since $x_{2}^{*}-x_{1}<2 m$, we either have a "competitive" price equilibrium or none for $x_{1}-m<$ $z<x_{2}^{*}$. Let:

$$
\begin{aligned}
& z_{1}^{u}=\frac{1}{8}\left[3+18 m+4 x_{1}-6 \sqrt{2 m\left(12 m+4 x_{1}-3\right)}\right] \\
& z_{2}^{u}=\frac{1}{8}\left[3-30 m+4 x_{1}+6 \sqrt{2 m\left(12 m+4 x_{1}-3\right)}\right]
\end{aligned}
$$

and it follows that firm 1 never wants to undercut the candidate equilibrium price of the rival for $z<z_{1}^{u}$, while the opposite is true for $z>z_{2}^{u}\{24\}$. 
1. Consider first the case of $x_{1} \in\left(x_{2}^{*}-2 m, x_{2}^{*}-m\right)$. We then have $\{25\}$ :

$$
x_{2}^{*}>z_{1}^{u}>x_{2}^{*}-m>x_{1}>z_{2}^{u}>x_{1}-m
$$

which means the price equilibrium does not exist for $z \in\left(x_{1}-m, z_{2}^{u}\right)$ and for $z \in$ $\left(z_{1}^{u}, x_{2}^{*}\right)$. Since $z_{1}^{u}>x_{1}-m / 2\{26\}$, the best the deviating player can get in the latter case is $\bar{\pi}_{r}$. As for $z \in\left(x_{1}-m, z_{2}^{u}\right)$, let $\widehat{x_{1}}=3 / 4+(5 / 2-3 \sqrt{2}) m$ and observe that $x_{1}<\widehat{x_{1}} \Leftrightarrow z_{2}^{u}<x_{1}-m / 2\{27\}$. Consequently:

(a) for $x_{1} \in\left(x_{2}^{*}-2 m, \widehat{x_{1}}\right)$, the best the deviating player can get when $z \in\left(x_{1}-m, z_{2}^{u}\right)$ is $\bar{\pi}_{l}$ and the maximum expected profit becomes:

$$
\widehat{\Pi}_{1}^{a}=\frac{1}{1-m}\left[\int_{0}^{x_{1}-m}\left(x_{2}^{*}-x_{1}\right) d z+\int_{x_{1}-m}^{z_{2}^{u}} \bar{\pi}_{l} d z+\int_{z_{2}^{u}}^{z_{1}^{u}} \pi_{c}^{*} d z+\int_{z_{1}^{u}}^{x_{2}^{*}} \bar{\pi}_{r} d z\right]
$$

(b) for $x_{1} \in\left(\widehat{x_{1}}, x_{2}^{*}-m\right)$, the best the deviating player can get is $\bar{\pi}_{l}$ when $z \in$ $\left(x_{1}-m, x_{1}-m / 2\right)$ and $\bar{\pi}_{r}$ when $z \in\left(x_{1}-m / 2, z_{2}^{u}\right)$, so that the maximum expected profit becomes:

$$
\widehat{\Pi}_{1}^{b}=\frac{1}{1-m}\left[\int_{0}^{x_{1}-m} x_{2}^{*}-x_{1} d z+\int_{x_{1}-m}^{x_{1}-\frac{m}{2}} \bar{\pi}_{l} d z+\int_{x_{1}-\frac{m}{2}}^{z_{2}^{u}} \bar{\pi}_{r} d z+\int_{z_{2}^{u}}^{z_{1}^{u}} \pi_{c}^{*} d z+\int_{z_{1}^{u}}^{x_{2}^{*}} \bar{\pi}_{r} d z\right]
$$

2. Suppose now $x_{1} \in\left(x_{2}^{*}-m, x_{2}^{*}\right)$ and let $\widetilde{x}_{1}=3 / 4-m$. We have $z_{1}^{u}<z_{2}^{u} \Leftrightarrow x_{1}>$ $\widetilde{x}_{1}\{28\}$ and:

(a) for $x_{1} \in\left(x_{2}^{*}-m, \widetilde{x}_{1}\right)$ we have $x_{1}-m / 2<z_{2}^{u}<z_{1}^{u}<x_{2}^{*}\{29\}$, so that the maximum expected profit is still given by $\widehat{\Pi}_{1}^{b}$.

(b) for $x_{1} \in\left(\widetilde{x}_{1}, x_{2}^{*}\right)$ we have $z_{2}^{u}>z_{1}^{u}$, so that no "competitive" equilibria exist and the maximum expected profit becomes:

$$
\widehat{\Pi}_{2}^{b}=\frac{1}{1-m}\left[\int_{0}^{x_{1}-m} x_{2}^{*}-x_{1} d z+\int_{x_{1}-m}^{x_{1}-\frac{m}{2}} \bar{\pi}_{l} d z+\int_{x_{1}-\frac{m}{2}}^{x_{2}^{*}} \bar{\pi}_{r} d z\right]
$$

All in all, in order to ensure that no deviation from $x_{1}^{*}$ is profitable, we need:

$$
\begin{aligned}
& \forall x_{1} \in\left(x_{2}^{*}-2 m, \widehat{x_{1}}\right): \Pi^{*} \geq \widehat{\Pi}_{1}^{a} \\
& \forall x_{1} \in\left(\widehat{x_{1}}, \widetilde{x}_{1}\right): \Pi^{*} \geq \widehat{\Pi}_{1}^{b} \\
& \forall x_{1} \in\left(\widetilde{x}_{1}, x_{2}^{*}\right): \Pi^{*} \geq \widehat{\Pi}_{2}^{b}
\end{aligned}
$$


and it can be shown $\{30\}$ that the conditions (C1) - C3 are all true for $m<\bar{m} \approx 1 / 9$ and $r \in\left[x_{2}^{*}, \phi(m)\right]$, where $\lim _{m \rightarrow 0} \phi(m)=+\infty$. By symmetry, the same conditions guarantee that no deviation from $x_{2}^{*}$ is profitable for player 2 .

It remains to recall that by Theorem 3 in Dasgupta and Maskin [8], a mixed-strategy price equilibrium exists in a Hotelling game with linear costs for any $x_{1}<x_{2}$. Clearly, the expected profit of a firm in any such equilibrium cannot exceed the optimal monopoly profit $\left(\bar{\pi}_{l}\right.$ or $\left.\bar{\pi}_{r}\right)$. Suppose the players play the pure-strategy equilibrium prices where available and mixed-strategy equilibrium prices otherwise. Then the first-stage expected profit of a player deviating from $x_{i}^{*}$ cannot exceed the one based on getting pure-strategy equilibrium profits where available and the optimal monopoly profits otherwise. Hence, the subgame-perfect equilibrium as described in the Proposition exists and has locations (2). 


\section{References}

[1] Philippe Aghion, Maria Paz Espinosa, and Bruno Jullien. Dynamic duopoly with learning through market experimentation. Economic Theory, 3(3):517-537, 1993.

[2] Simon P. Anderson. Equilibrium existence in the linear model of spatial competition. Economica, 55(220):479-491, 1988.

[3] Ronald Balvers and Lazlo Szerb. Location in the hotelling duopoly model with demand uncertainty. European Economic Review, 40(7):1453-1461, 1996.

[4] Helmut Bester, Andre de Palma, Wolfgang Leininger, Jonathan Thomas, and ErnstLudwig von Thadden. A noncooperative analysis of hotelling's location game. Games and Economic Behavior, 12(2):165 - 186, 1996.

[5] Steffen Brenner. Hotelling games with three, four, and more players. Journal of Regional Science, 45(4):851-864, 2005.

[6] Javier F. Casado-Izaga. Location decisions: The role of uncertainty about consumer tastes. Journal of Economics, 71(1):31-46, 2000.

[7] Partha Dasgupta and Eric Maskin. The existence of equilibrium in discontinuous economic games, i: Theory. The Review of Economic Studies, 53(1):1-26, 1986.

[8] Partha Dasgupta and Eric Maskin. The existence of equilibrium in discontinuous economic games, ii: Applications. The Review of Economic Studies, 53(1):27-41, 1986 .

[9] C. d'Aspremont, J. Jaskold Gabszewicz, and J. F. Thisse. On hotelling's "stability in competition". Econometrica, 47(5):1145-1150, 1979.

[10] Nicholas Economides. The principle of minimum differentiation revisited. European Economic Review, 24(3):345 - 368, 1984.

[11] Nicholas Economides. Minimal and maximal product differentiation in hotelling's duopoly. Economics Letters, 21(1):67-71, 1986.

[12] J. Jaskold Gabszewicz and J.-F. Thisse. On the nature of competition with differentiated products. The Economic Journal, 96(381):160-172, 1986.

[13] Jean J. Gabszewicz and Jacques-Francois Thisse. Location. volume 1 of Handbook of Game Theory with Economic Applications, chapter 9, pages 281 - 304. Elsevier, 1992.

[14] John F. R. Harter. Hotelling's competition with demand location uncertainty. International Journal of Industrial Organization, 15(3):327-334, 1997. 
[15] Harold Hotelling. Stability in competition. The Economic Journal, 39(153):41-57, 1929.

[16] Phillip J. Lederer and Jr. Hurter, Arthur P. Competition of firms: Discriminatory pricing and location. Econometrica, 54(3):623-640, 1986.

[17] Kieron J. Meagher and Klaus G. Zauner. Product differentiation and location decisions under demand uncertainty. Journal of Economic Theory, 117(2):201-216, 2004.

[18] Kieron J. Meagher and Klaus G. Zauner. Location-then-price competition with uncertain consumer tastes. Economic Theory, 25(4):799-818, 2005.

[19] Pierre R. Merel and Richard J. Sexton. Kinked-demand equilibria and weak duopoly in the hotelling model of horizontal differentiation. The B.E. Journal of Theoretical Economics, 10: Iss. 1 (Contributions), 2010.

[20] Martin J. Osborne and Carolyn Pitchik. Equilibrium in hotelling's model of spatial competition. Econometrica, 55(4):911-922, 1987. 